\title{
Triple-Phase Shift Modulation for Dual Active Bridge based on Simplified Switching Loss Model
}

\author{
Simone Pistollato \\ Dept. of Management and Engineering \\ University of Padova \\ Vicenza, Italy \\ simone.pistollato@phd.unipd.it
}

\author{
Tommaso Caldognetto \\ Dept. of Management and Engineering \\ University of Padova \\ Vicenza, Italy \\ tommaso.caldognetto@unipd.it \\ Paolo Magnone \\ Dept. of Management and Engineering \\ University of Padova \\ Vicenza, Italy \\ paolo.magnone@unipd.it
}

\author{
Paolo Mattavelli \\ Dept. of Management and Engineering \\ University of Padova \\ Vicenza, Italy \\ paolo.mattavelli@unipd.it
}

\begin{abstract}
This paper proposes and analyzes triple phase shift modulation techniques aiming at maximizing the efficiency of the dual active bridge (DAB) converter. The DAB converter finds natural application in smart dc power systems integrating renewables and energy storage systems, for which loss minimization is crucial. This paper shows that the investigated modulation schemes allow converter efficiency improvements by exploiting complete or partial zero-voltage switching in a range of operating conditions as wide as possible, similarly to other papers in the literature based on triple phase shift modulation. Distinctively, the approach presented herein is based on simple converter modeling and the characterization of the switching elements. Experimental results are reported showing the performance attained by the proposed techniques considering a $1.5-\mathrm{kW}$ converter prototype.

Index Terms-dc-dc converters; dual active bridge; efficiency; loss minimization; triple phase shift; zero-voltage switching.
\end{abstract}

\section{INTRODUCTION}

Nowadays, dc renewable energy sources and energy storage systems are gaining ground in many application fields. Such resources are often interconnected through electronic power converters (EPC) to form nano- or micro- grids [1], as shown in Fig. 1. Similar structures can be found in different application scenarios, like, for example, in the implementation of the electrical powertrain of hybrid or electric vehicles [2], [3]. Typically, these energy resources provide a non-regulated output voltage, varying with their status (e.g., the state of charge for a battery storage system or the irradiation level for a photovoltaic array), and thus require suitable EPCs to interface with a common distribution bus of known nominal voltage. EPCs process the energy from the resources and supply loads at specific voltage levels, therefore, an optimal operation of the EPCs is crucial to ensure an efficient and reliable operation of the whole system.

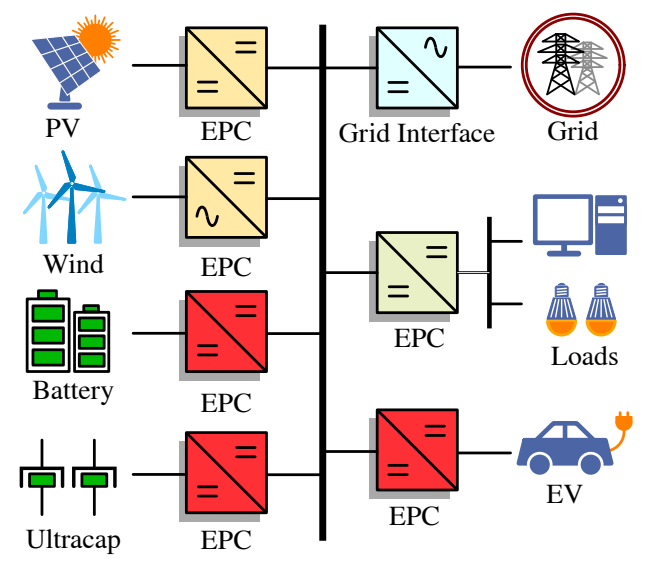

Fig. 1: Electronic power converters (EPC) in a dc nanogrid.

Several dc-dc converter topologies, with different characteristics in terms of step-up ratios, power density, switching and conduction losses, power flow direction, galvanic isolation, and control performance, are described in the literature [4]. The dual active bridge (DAB) [5] is one of the most renown for implementing isolated bidirectional converters (the red blocks in Fig. 1), on which this paper focuses.

A comprehensive operation and control analysis of the DAB can be found in [6]. The studies reported in the literature mainly concern efficiency and performance improvements, which are addressed in different ways. Two main issues affect the DAB performance: the increase of the transformer's circulating currents when the input-output voltage ratio is different from the transformer's turns ratio, which brings to increased rms currents and conduction losses [7]; the loss of zero voltage switching (ZVS) turn-on in specific operating conditions, especially at light load [8]. Various modulation strategies aiming at minimizing the conduction losses and ensuring ZVS operation are reported. The Phase Shift Modu- 
lation (PSM) [6] is a simple, classical modulation technique by which the DAB topology can achieve ZVS over a range of operating conditions that depends on the input-output voltage ratio and the transferred power [7]. Improvements can be achieved by more advanced modulation techniques, like the extended phase-shift (EPS) [9], the dual phase-shift (DPS) [10], and the triple phase-shift (TPS) [11], which better exploit the available degrees of freedom of the topology. Based on a TPS approach, modulation strategies aiming at conduction loss minimization and efficiency maximization are proposed in [12] and [13], respectively. Both modulations rely on accurate and precise models of the converter operation and loss contributions [14], which might be not easily available in practical implementations and pose deployment challenges in digital control platforms.

The mentioned efficiency improvement aspects are tackled herein by triple phase modulation techniques that aim at ZVS operation of the converter, so that to reduce the significant amount of power dissipation related to the switching losses. This is achieved by an analysis and also a simple characterization of the converter in order to identify convenient, in terms of efficiency, triple phase shift modulations.

In the following, a formal assessment of the topology operation is reported in Sect. II. Sect. III presents the main loss contributions affecting the efficiency of the topology, reviewing the desirable features of candidate modulation techniques for minimizing converter losses. On the basis of the obtained analysis results and models, different approaches for selecting convenient modulation parameters are presented in Sect. IV. It is shown that the resulting triple-phase shift modulation maps allow to improve the converter efficiency with respect to the classically used modulations, that is the PSM, especially at light load. The obtained results are finally experimentally verified in Sect. V, also reporting the performance measured on a prototype rated for $1.5 \mathrm{~kW}$.

\section{DAB CHARACTERISTICS AND OPERATION}

A basic DAB topology is displayed in Fig. 2a. It consists of a high-frequency transformer and two full bridges connected at the primary (i.e, low-voltage) and secondary (i.e., highvoltage) sides. The transformer allows galvanic isolation and high step-up/down ratios between the two sides; its leakage inductance, possibly combined with an additional external inductor, is exploited as energy transfer element. The full bridges can impress three-level voltage waveforms $v_{A}$ and $v_{B}$, defining the power flow intensity and direction. An equivalent representation is provided in Fig. $2 \mathrm{~b}$, where $v_{A}$ and $v_{B}$, by appropriate modulation schemes, present patterns achieving the desired power flow and switches operation. A classical modulation technique is the PSM, in which $v_{A}$ and $v_{B}$ are fixed-frequency square waveforms whose relative phase-shift is adjusted to control the power flow between the two sides [6], as shown in Fig. 3. By using the EPS, DPS, and TPS modulations, it is possible to control the pulse duration of $v_{A}$, $v_{B}$, or both. These additional degrees of freedom are exploited to improve the conversion efficiency, as discussed in Sect. IV.

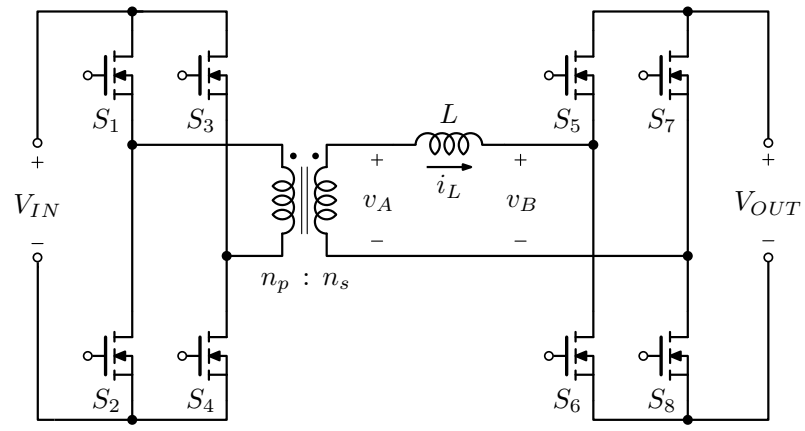

(a)

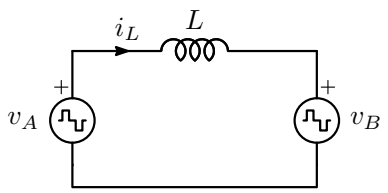

(b)

Fig. 2: (a) Dual Active Bridge (DAB) converter topology; (b) equivalent circuit for power transfer analysis.

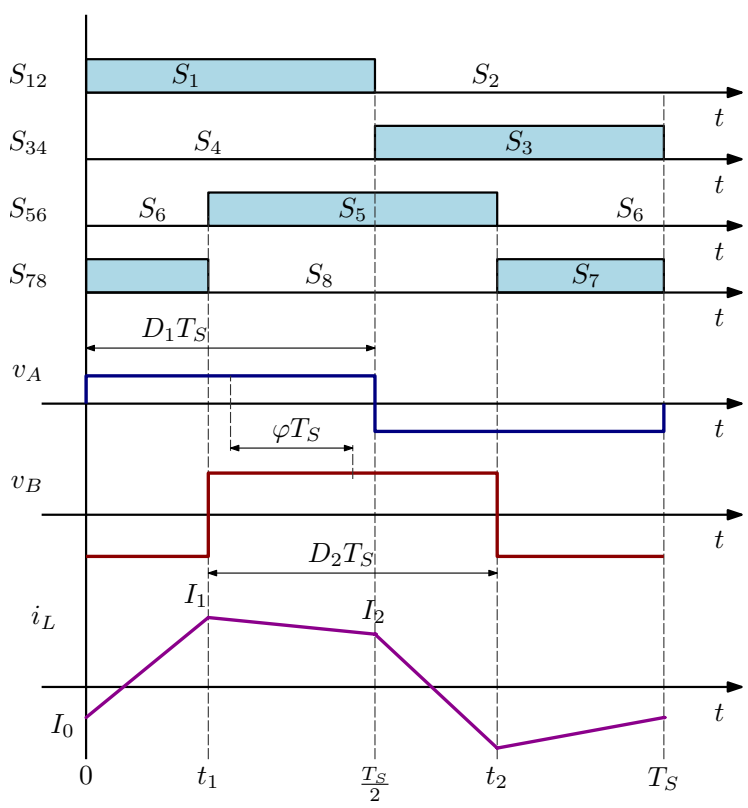

Fig. 3: Phase-shift modulation (PSM): the input and output bridges operate with $50 \%$ duty-cycle $D_{1}$ and $D_{2}$, while the phase shift $\varphi$ is adjusted to modulate the power transfer.

\section{CONVERTER LOSSES}

The considered loss contributions include the conduction losses due to the on-resistance of the switches [7], the losses due to the windings resistances of the transformer [14], and the switching losses [15]. Other contributions like driving circuit losses, magnetic core losses, and other frequencydependent losses, also related to the printed circuit board, are not analytically modeled herein. 
The conduction loss for the DAB topology is modeled as:

$$
\begin{array}{r}
P_{\text {cond }}=2 R_{\text {on }, L V}\left(n I_{L, r m s}\right)^{2}+2 R_{o n, H V} I_{L, r m s}^{2}+ \\
R_{\text {pri }}\left(n I_{L, r m s}\right)^{2}+R_{\text {sec }} I_{L, r m s}^{2}
\end{array}
$$

where $R_{o n, L V}$ is the on-resistance of the low-voltage switches, $R_{o n, H V}$ is the on-resistance of the high-voltage switches, $R_{p r i}$ is the resistance of the low-voltage side winding, $R_{s e c}$ is the resistance of the high-voltage side winding, $n$ is the turns ratio (see Table I) and $I_{L, r m s}$ the rms current value of the equivalent energy transfer inductance $L$.

For what concerns the switching losses, these depend, mainly, on the switches' equivalent drain-source capacitance, the chosen dead-times, the value of inductor $L$ and the stored energy at the switching instants, and the switching frequency. An accurate model of the switching losses is derived in [16] and can be estimated as described in [14]. An effective means to minimize such a loss contribution is to ensure ZVS [16] at the turn-on. Based on the circuit in Fig. 4a, hard switching (HS) occurs when $S_{2}$ turns on with a voltage $v_{C}$ close to $V_{x}$ (Fig. 4b): the beginning of the plateau region of $v_{g s, S_{2}}$ coincides with the transition of the voltage $v_{C}$ toward zero. In this case the equivalent capacitance at node $C$ is completely discharged by means of $S_{2}$, resulting in a dissipative process and the worst condition in terms of losses. On the contrary, with ZVS, the switching loss is minimum if $S_{2}$ turns on when $v_{C}$ has already reached zero, as in Fig. $4 \mathrm{~d}$; in this case only the inductor current $i_{L}$ (if of appropriate sign) determines the transition of the node voltage $v_{C}$ toward zero, by means of a non-dissipative process where the energy is exchanged with the inductor. All the intermediate conditions where the switch $S_{2}$ turns on during the transition of $v_{C}$ toward zero are called partial ZVS, represented in Fig. 4c. Similar considerations can be done for the opposite transition of $v_{C}$.

\section{A. Minimum Current Constraint}

On this light, considering a fixed deadtime between the turnoff of a switch and the turn-on of the complementary one, ZVS can be achieved by ensuring a sufficiently intense current of appropriate sign at the beginning of the deadtime, that is, in the case of Fig. 4, at the turn-off of $S_{1}$. To a first approximation, assuming a constant inductor current during the deadtime, the ZVS condition translates into a minimum current constraint:

$$
I_{S_{x}, \text { turn-on }}>I_{S_{x}, \text { turn-on }}^{\min }=\frac{C_{e q, Q} V_{i}}{t_{\text {dead }}}
$$

where $C_{e q, Q}$ is the equivalent charge capacitance [15] at the node, $V_{i}$ is its voltage at the switching instant (i.e., $V_{I N}$ or $\left.V_{O U T}\right)$, and $t_{\text {dead }}$ is the dead-time. Remarkably, switching losses typically account for a predominant portion of the total converter losses in the considered application [12].

A first modulation approach could be to chose the dutycycles and phase-shift of the converter to satisfy (2). Of course, this may be a too hard constraint that is not possible to achieve in particular operating conditions. However in such cases partial ZVS may still be possible.

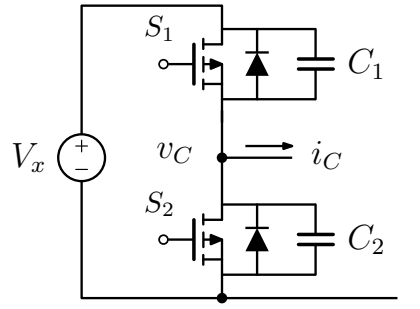

(a)

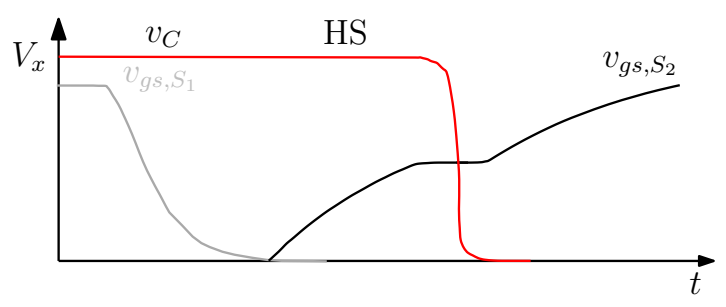

(b)

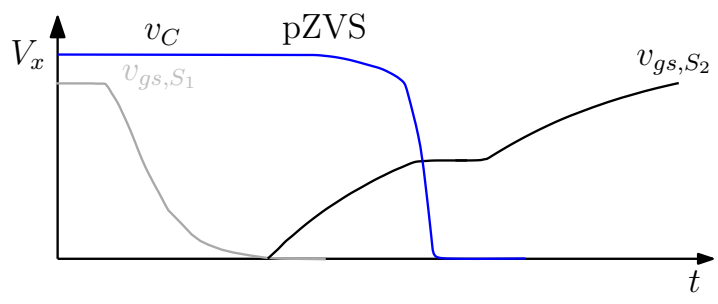

(c)

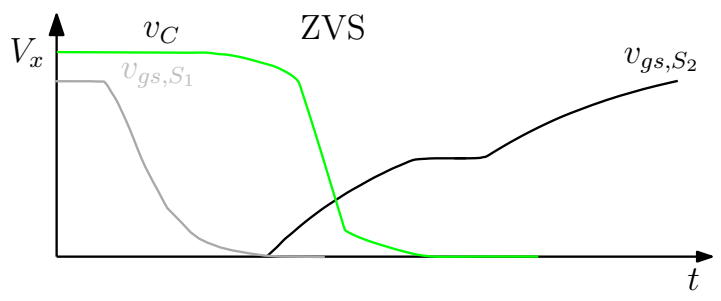

Fig. 4: Typical behavior across the turn-off of upper switch $S_{1}$ and the turn-on of lower switch $S_{2}$. From top to bottom: hard switching (red), partial ZVS (blue), and ZVS (green).

\section{B. Switching Leg Behavior Characterization}

To exploit partial ZVS the model introduced in the previous paragraph should be refined in order to suitably describe the behavior of the node voltage $v_{C}$ along the transition from the full voltage to zero and vice-versa. Due to the highly nonlinear behavior, the waveforms of $v_{C}$ and $i_{C}$ are acquired during a ZVS of the kind in Fig. $4 \mathrm{~d}$ and the charge exchange giving a ZVS, a partial ZVS or a hard switching transition extrapolated from the measurements.

By referring to the experimental prototype considered in this paper and described in Sect. V, a switching transition from $50 \mathrm{~V}$ to $0 \mathrm{~V}$ of the drain source voltage of switch $S_{2}$ (the central node of $S_{12} \mathrm{leg}$ ) is used to characterize the input switches behavior (Fig. $5 \mathrm{a}$ ), while $400 \mathrm{~V}$ to $0 \mathrm{~V}$ of switch $S_{6}$ (the central node of $S_{56} \mathrm{leg}$ ) to characterize the output ones (Fig. 5b). By using these waveforms it is possible to derive the charge $Q_{\text {node }}$ necessary to discharge the node $C$ from a certain voltage value to zero and also the losses $P_{s w}$. In 


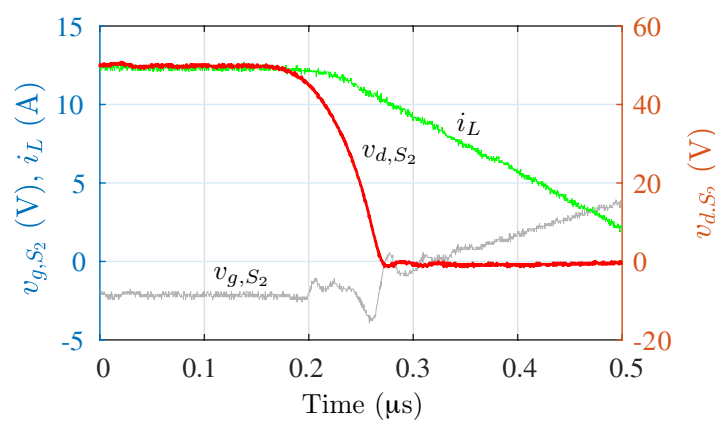

(a)

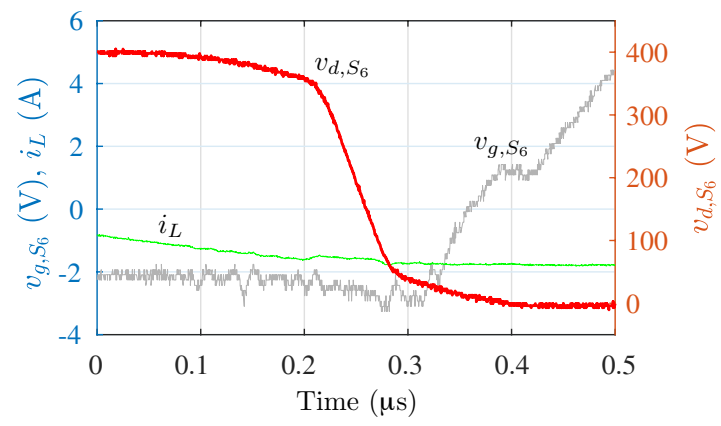

(b)

Fig. 5: Measured switching waveforms at the turn-on of $S_{2}$ (a) and $S_{6}$ (b).

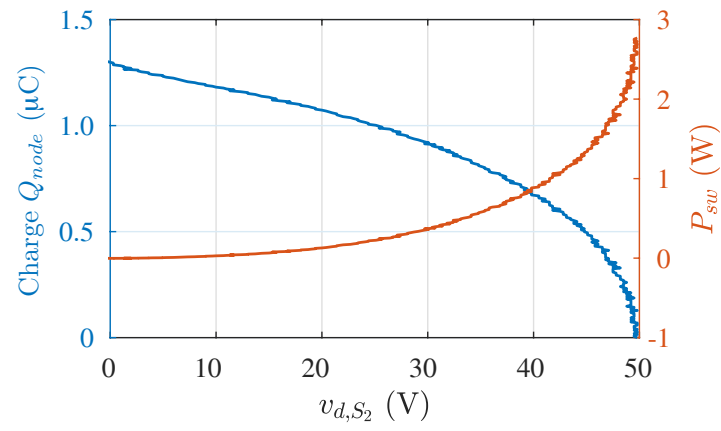

(a)

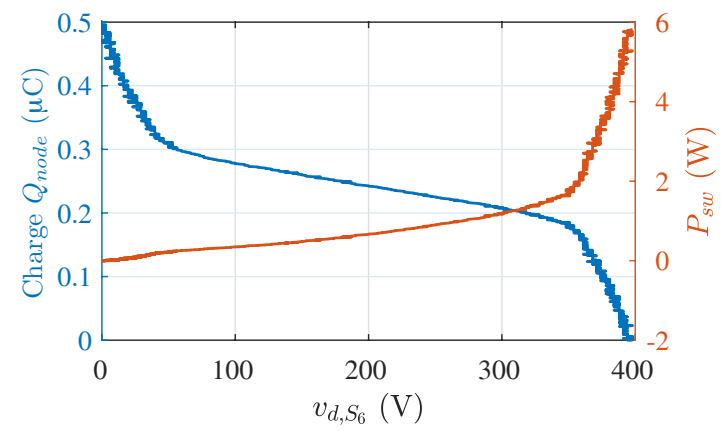

(b)

Fig. 6: $Q_{\text {node }}$ and $P_{s w}$ as a function of drain-source voltage of $S_{2}$ (a) and $S_{6}$ (b). $P_{s w}$ values are calculated with a switching frequency of $60 \mathrm{kHz}$ (see Table. I). Relations derived from the measurements reported in Fig. 5.
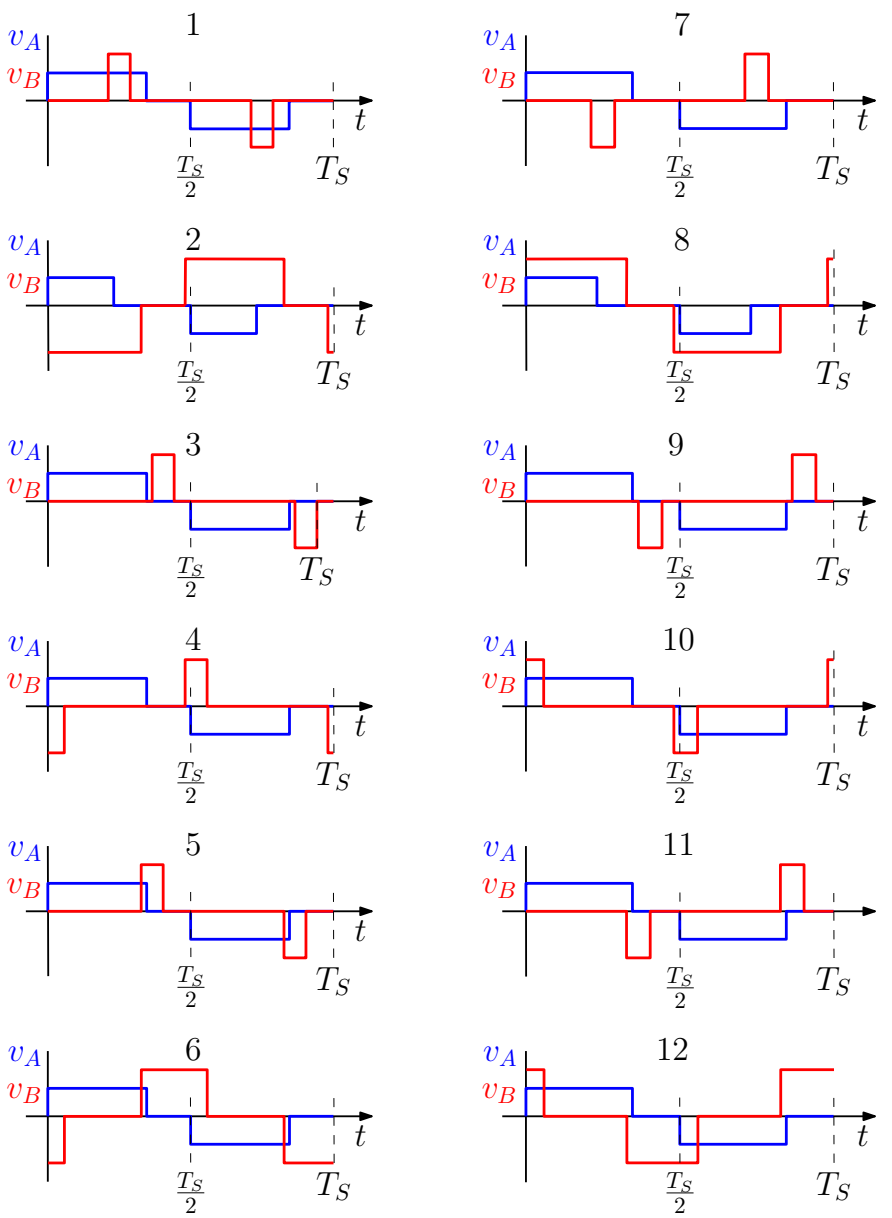

Fig. 7: Possible switching sequences for $v_{A}$ and $v_{B}$ in Fig. $2 \mathrm{~b}$.

Fig. 6a and Fig. $6 \mathrm{~b}$ the charge and the losses, expressed as a function of the drain-source voltage of the input switches $S_{2}$ and $S_{6}$, respectively, are presented. Similar considerations can be done for the complementary switches $S_{1}$ and $S_{5}$, obtaining the complete behavior of the input bridge legs $S_{12}, S_{56}$ and of the output bridge legs $S_{34}, S_{78}$, by exploiting the symmetry of the DAB structure.

\section{TPS MODUlation SCHEMES}

In Fig. 7 are presented all the possible switching sequences, categorized by the order of the switching edges of voltages $v_{A}$ and $v_{B}$ of Fig. 2b [11], that are achievable with TPS. Twelve cases result, for which it is possible to derive the instantaneous behavior of the inductor current $i_{L}$, which gives information on the rms current values linked to conduction losses and on the current values at the switching instants to recognize ZVS conditions [15].

On the basis of the considerations reported in Sect. III and the complete converter analysis covering all the cases in Fig. 7, three approaches are investigated herein to define triplets $D_{1}$, $D_{2}$, and $\varphi$ (defined in Fig. 3) that, for a given power transfer $P_{O U T}$ and input and output voltage $V_{I N}$ and $V_{O U T}$, provide improved converter operation: 


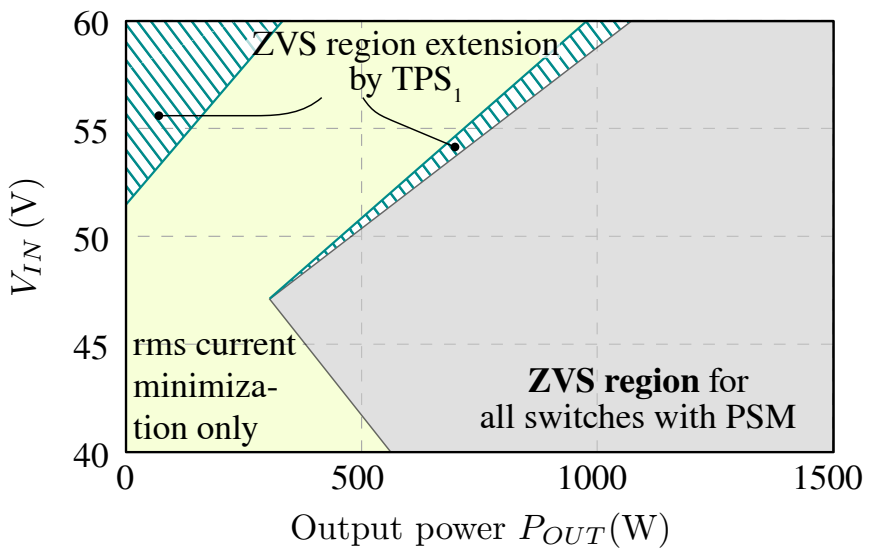

Fig. 8: ZVS regions for DAB by using simulation models. Grey regions relate to the PSM, tiling-pattern areas are the additional ZVS regions attained with first approach TPS $_{1}$.

1) First approach $\left(\mathrm{TPS}_{1}\right)$ : select, for a given power transfer, triplets that primarily favor ZVS conditions and, for equal ZVS conditions, sequences presenting minimum rms current.

2) Second approach $\left(\mathrm{TPS}_{2}\right)$ : select, for a given power transfer, triplets that primarily favor ZVS conditions at least at the input or the output bridge, if not possible for both, and, for equal ZVS conditions, sequences presenting minimum rms current.

3) Third approach $\left(\mathrm{TPS}_{3}\right)$ : select, for a given power transfer, triplets that primarily favor ZVS conditions and, for equal ZVS conditions, sequences presenting minimum rms current. For non-ZVS conditions (partial ZVS) select sequences that ensure minimum switching losses and present minimum rms current.

In all the cases, rms current minimization only is considered in case it is not possible to exploit ZVS or partial ZVS at the input or at the output. The first approach uses the minimum current constraint (2) in Sect. III-A to aim at full ZVS of the input and output bridge, with ZVS regions displayed in Fig. 8 together with the classical modulation scheme. Notably, the ZVS region is extended by this approach, leading, as shown in Sect. V, to lower total losses. The second approach uses the same minimum current constrain and is an evolution of the first one because it considers not only the complete ZVS but also favor, if not complete ZVS is achieved, the ZVS of all the input bridge or output bridge switches. The map in Fig. 9 refers to this latter scheme. Notably, the ZVS regions of the input and output bridge are the same of the previous scheme, but ZVS for the input bridge is also achieved at light load. The last approach relaxes the constraints by exploiting also partial ZVS conditions, that is, all the intermediate conditions between ZVS and hard switching, as discussed in Sect. III-B. Fig. 10 displays the values of $D_{1}, D_{2}$, and $\varphi$ with respect to the output power level using this third approach.

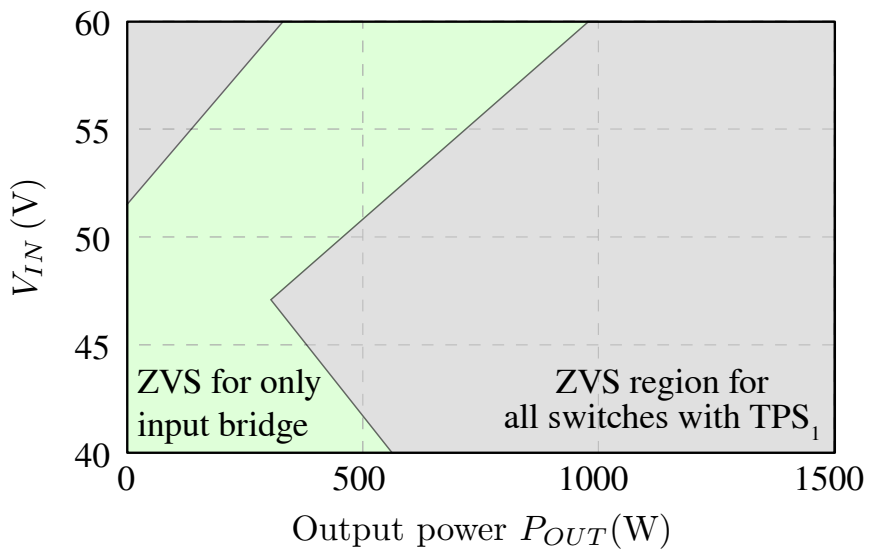

Fig. 9: ZVS regions by using simulation models with second approach $\mathrm{TPS}_{2}$. Grey regions relate to the complete ZVS, while the light green one to the ZVS of the input switches.

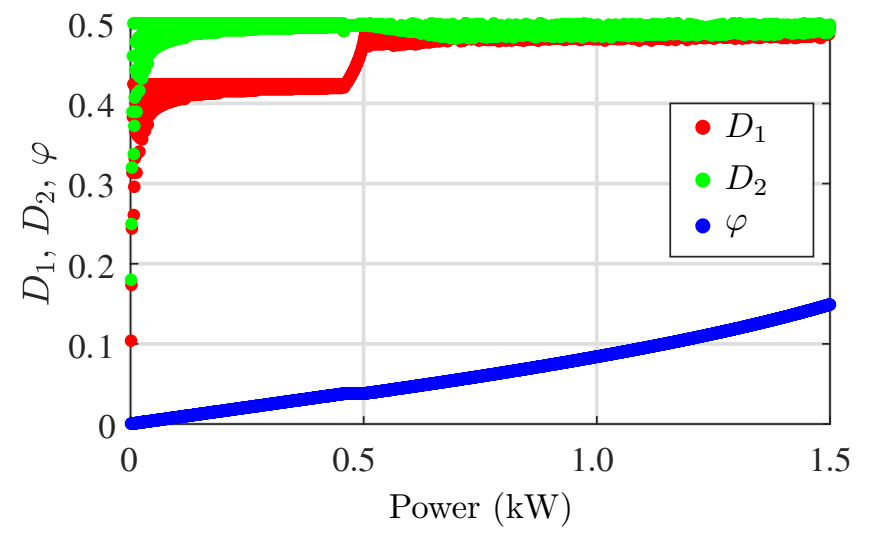

Fig. 10: Sequences of $D_{1}, D_{2}$, and $\varphi$ according the particular operating condition $V_{I N}=50 \mathrm{~V}, V_{O U T}=400 \mathrm{~V}$ with third approach $\mathrm{TPS}_{3}$ by using simulation models.

\section{EXPERIMENTAL RESULTS}

This section reports some preliminary experimental results considering a DAB converter prototype rated for $1.5 \mathrm{~kW}$. The parameters of the converter are listed in Table I. The converter is connected to a power supply, at the input port, and to an electronic load, at the output port. A LAUNCHXLF28377S development board with a TI TMS320F28377S DSP embedded is used to implement the modulators and generate the PWM signals.

Fig. 11 shows the efficiency curves obtained with the investigated approaches, together with the efficiency obtained with the classical PSM at the nominal conditions of $V_{I N}=$ $50 \mathrm{~V}, V_{O U T}=400 \mathrm{~V}$. Higher efficiencies can be obtained with respect to the PSM with all the investigated approaches, the maximum recorded improvements, amount to $1.2 \%$ with

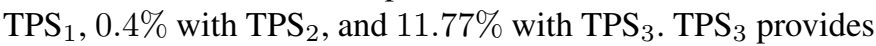
significant improvements thanks to a more precise modeling of the switching losses, also during partial ZVS. 
TABLE I: DAB Converter parameters (Fig. 2a)

\begin{tabular}{|c|c|}
\hline Parameters & \\
\hline Input voltage $V_{I N}$ & $40 \div 60$ \\
\hline Output voltage $V_{O U T}$ & 400 \\
\hline Nominal power $P_{N} \quad(\mathrm{~kW})$ & 1.5 \\
\hline Switching frequency $f_{s}=\frac{1}{T_{s}}(\mathrm{kHz})$ & 60 \\
\hline Energy transfer inductance $\quad(\mu \mathrm{H})$ & 186 \\
\hline Turns ratio $\left(n=n_{s} / n_{p}\right)$ & 8 \\
\hline Low-voltage side devices & IPP023N10N5 \\
\hline High-voltage side devices & FCP110N65F \\
\hline
\end{tabular}

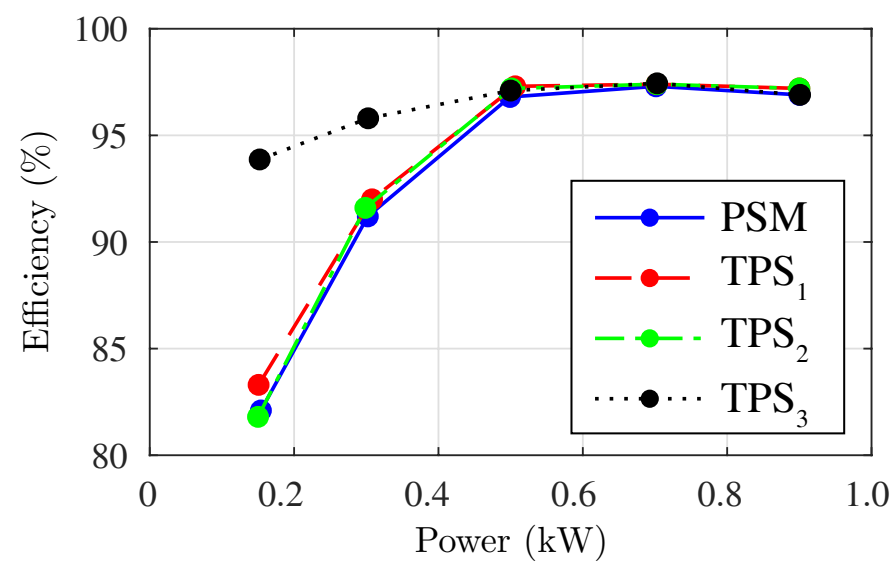

Fig. 11: Efficiency curves at $V_{I N}=50 \mathrm{~V}, V_{O U T}=400 \mathrm{~V}$.

\section{CONCLUSION}

In this paper the dual active bridge (DAB) is analyzed and three modulation approaches are proposed and tested to improve the converter's efficiency. Zero-voltage switching maps are reported to show the most favorable operating conditions to reduce switching and conduction losses contributions. The results are validated considering an experimental DAB converter prototype. It is shown that accounting ZVS with the characterization of switching behavior of the devices allows significant improvements with respect to simply give a constraint on the instantaneous current switching values, at the reported operating conditions.

\section{ACKNOWLEDGMENT}

The authors would like to thank the financial support from the Electronic Components and Systems for European Leadership Joint Undertaking under grant agreement No 737434. This Joint Undertaking receives support from the European Unions Horizon 2020 research and innovation programme and Germany, Slovakia, Netherlands, Spain, Italy.

\section{REFERENCES}

[1] G. Liu et al., "Plug and Play DC-DC Converters for Smart DC Nanogrids with Advanced Control Ancillary Services," in 2018 IEEE 23rd International Workshop on Computer Aided Modeling and Design of Communication Links and Networks (CAMAD), Sep 2018, pp. 1-6.

[2] M. Carignano et al., "Assessment of Energy Management in a Fuel Cell/Battery Hybrid Vehicle," vol. 7, 2019, pp. 16 110-16122.
[3] G. Buticchi et al., "On-Board Microgrids for the More Electric Aircraft-Technology Review," IEEE Trans. Ind. Electron., vol. 66, no. 7, pp. 5588-5599, Jul 2019.

[4] M. Forouzesh et al., "Step-Up DC-DC Converters: A Comprehensive Review of Voltage-Boosting Techniques, Topologies, and Applications," vol. 32, no. 12, Dec 2017, pp. 9143-9178.

[5] B. Zhao et al., "Overview of Dual-Active-Bridge Isolated Bidirectional DC-DC Converter for High-Frequency-Link Power-Conversion System," vol. 29, no. 8, Aug 2014, pp. 4091-4106.

[6] C. Mi et al., "Operation, design and control of dual H-bridge-based isolated bidirectional DC-DC converter," vol. 1, no. 4, Dec 2008, pp. 507-517.

[7] V. M. Iyer et al., "Optimal design methodology for dual active bridge converter under wide voltage variation," in 2017 IEEE Transportation Electrification Conference and Expo (ITEC), Jun 2017, pp. 413-420.

[8] G. Xu et al., "Fixed duty cycle compensation and magnetizing current design for DAB DC-DC converter with conventional trapezoidal modulation to achieve zero voltage switching," in 2016 IEEE 8th International Power Electronics and Motion Control Conference (IPEMCECCE Asia), May 2016, pp. 433-438.

[9] B. Zhao et al., "Extended-Phase-Shift Control of Isolated Bidirectional DC-DC Converter for Power Distribution in Microgrid," vol. 27, no. 11, Nov 2012, pp. 4667-4680.

[10] H. Bai et al., "Eliminate Reactive Power and Increase System Efficiency of Isolated Bidirectional Dual-Active-Bridge DC-DC Converters Using Novel Dual-Phase-Shift Control," vol. 23, no. 6, Nov 2008, pp. 29052914.

[11] Y. A. Harrye et al., "Comprehensive steady state analysis of bidirectional dual active bridge DC/DC converter using triple phase shift control," in 2014 IEEE 23rd International Symposium on Industrial Electronics (ISIE), Jun 2014, pp. 437-442.

[12] F. Krismer et al., "Closed Form Solution for Minimum Conduction Loss Modulation of DAB Converters," vol. 27, no. 1, Jan 2012, pp. 174-188.

[13] — , "Efficiency-Optimized High-Current Dual Active Bridge Converter for Automotive Applications," vol. 59, no. 7, Jul 2012, pp. 27452760.

[14] _ _ "Accurate Power Loss Model Derivation of a High-Current Dual Active Bridge Converter for an Automotive Application," vol. 57, no. 3, Mar 2010, pp. 881-891.

[15] D. Costinett et al., "Circuit-oriented modeling of nonlinear device capacitances in switched mode power converters," in 2012 IEEE 13th Workshop on Control and Modeling for Power Electronics (COMPEL), Jun 2012, pp. 1-8.

[16] M. Kasper et al., "ZVS of Power MOSFETs Revisited," vol. 31, no. 12, Dec 2016, pp. 8063-8067. 